\title{
UN MODELO GRAVIMÉTRICO 3D DE LA PROFUNDA CUENCA SEDIMENTARIA DE ISCHIGUALASTO-VILLA UNIÓN (SAN JUAN Y LA RIOJA)-ARGENTINA.
}

\author{
F. Ruiz ${ }^{1} \&$ A. Introcaso ${ }^{2}$
}

\begin{abstract}
Se efectuaron relevamientos gravimétricos areales que cubren la profunda cuenca intermontana de Ischigualasto-Villa Unión y que abarcan el norte de la Precordillera Andina y una importante porción de las Sierras Pampeanas Occidentales. A partir de los datos observados se prepararon cartas de anomalías de Bouguer (AB) y de anomalías de Bouguer afectadas por corrección geológica (ABcg). La cuenca se ubica en un ambiente tectónico complejo que contamina fuertemente el campo gravimétrico. El presente estudio apunta a interpretar la geometría 3D de la cuenca sedimentaria mediante análisis de los datos gravimétricos. La separación del efecto gravimétrico del depósito sedimentario es una tarea compleja que se resolvió empleando: $\mathrm{i}$ - el método de suavizado gráfico sobre la $\mathrm{AB}$ y ii- filtrados numéricos sobre la $\mathrm{ABcg}$ ( $\mathrm{AB}$ corregida por los efectos sedimentarios de cuencas vecinas a la estudiada). Este análisis permite inferir que en el centro y norte de la cubeta las anomalías gravimétricas se deben, principalmente, al efecto del paquete sedimentario, mientras que en el sur de la misma, prevalecen efectos positivos en corteza debidos, muy probablemente, a intrusiones de rocas máficas. Por inversión gravimétrica de la anomalía residual se preparó un modelo gravimétrico, apoyado en datos sísmicos, que proporciona potencias sedimentarias de hasta 9 $\mathrm{km}$ en la región central de la cuenca.
\end{abstract}

Palabras Clave: Cuenca sedimentaria; Efecto gravimétrico; Filtrados; Modelo gravimétrico.

A 3D GRAVITY MODEL OF THE SEDIMENTARY DEEP BASIN OF ISCHIGUALASTOVILLA UNIÓN (SAN JUAN AND LA RIOJA), ARGENTINA- Areal gravimetric surveys were carried out in the Ischigualasto-Villa Unión intermontane deep basin, the north of the Andean Precordillera, and in a wide portion of the western Sierras Pampeanas. Maps of Bouguer anomalies $(A B)$ and Bouguer anomalies "affected by geological reduction" (ABcg) were derived from the observed data. The basin is located in a complex tectonic setting that strongly contaminates the gravity field. The present study aims to interpret the $3 D$ geometry of the sedimentary basin through gravity data analysis. The separation of the gravimetric effect of the sedimentary filling is a complex task presently solved by employing: $i$ - a graphical smoothing method from $A B$; and ii- numerical filterings from $A B c g$ ( $A B$ corrected by the sedimentary filling effect of neighboring basins). This analysis permits to deduce that in the center and north of the basin the gravity anomalies should be mainly the result of the sedimentary filling effect, whereas in the south of the basin positive effects in the crust prevail due most likely to mafic intrusive rocks. A gravity model supported by seismic data that provides sedimentary thicknesses of up to $9 \mathrm{~km}$ in the central region of the basin was formulated by using gravimetric inversion of the residual anomaly.

Key words: Sedimentary basin; Gravimetric effect; Filterings; Gravity model.

\footnotetext{
${ }^{1}$ CONICET y Facultad de Ciencias Exactas, Físicas y Naturales.

Universidad Nacional de San Juan. Meglioli 1160 (sur)

Rivadavia, C.P.: 5400, San Juan. Argentina.

Fax: (54) 064-234980, Tel.: (54) 064-340698

E-mail: fcoruiz@iinfo.unsj.edu.ar

${ }^{2}$ Facultad de Ciencias Exactas, Ingeniería y Agrimensura.

Universidad Nacional de Rosario. Instituto de Física de Rosario (CONICET).

Av. Pellegrini 250, (2000) Rosario, Argentina.
} 


\section{INTRODUCCIÓN}

Los Andes Centrales, desde $27^{\circ}$ a $33^{\circ} \mathrm{S}$, exhiben bloques de basamento flanqueados por fallas inversas (Sierras Pampeanas) al este de un cinturón de sobrecorrimientos epidérmicos (Pre-cordillera). Las Sierras Pampeanas producen un conjunto de cuencas de antepaís roto mientras que la Precordillera una cuenca de antepaís normal (Jordan, 1995). Esta comarca se caracteriza por la subducción subhorizontal de la placa de Nazca bajo la Sudamericana (Cahill \& Isacks, 1992).

La cuenca Ischigualasto-Villa Unión (I-VU) está ubicada en el ámbito geográfico de las Sierras Pampeanas Occidentales, en la región limítrofe con el norte de la Precordillera Andina (Fig. 1). Morfológicamente se trata de una depresión rodeada por sierras con diferentes características geológicas, que constituyen afloramientos de basamento cristalino, de rocas sedimentarias y volcánicas (De Alba, 1954). Comprende una extensa área, con más de $100 \mathrm{~km}$ en sentido meridiano y unos $50 \mathrm{~km}$ de ancho (Fig. 2), ocupada por sedimentos neopaleozoicos, triásicos y terciarios que configuran un amplio sinclinal con eje NNO-SSE (Stipanicic \& Bonaparte, 1979).

Georgieff (1992), Georgieff \& Ibañez (1994) y Malizia et al. (1995), publicaron resultados sísmicos parciales que señalan espesores de hasta $6 \mathrm{~km}$, para los sedimentos neógenos y, tal vez, más de $10 \mathrm{~km}$ teniendo en cuenta el total de los depósitos que rellenan la cubeta.

Con el objetivo de presentar un estudio integral de la

\section{MAPA DE UBICACION DE LA CUENCA ISCHIGUALASTO - VILLA UNION}

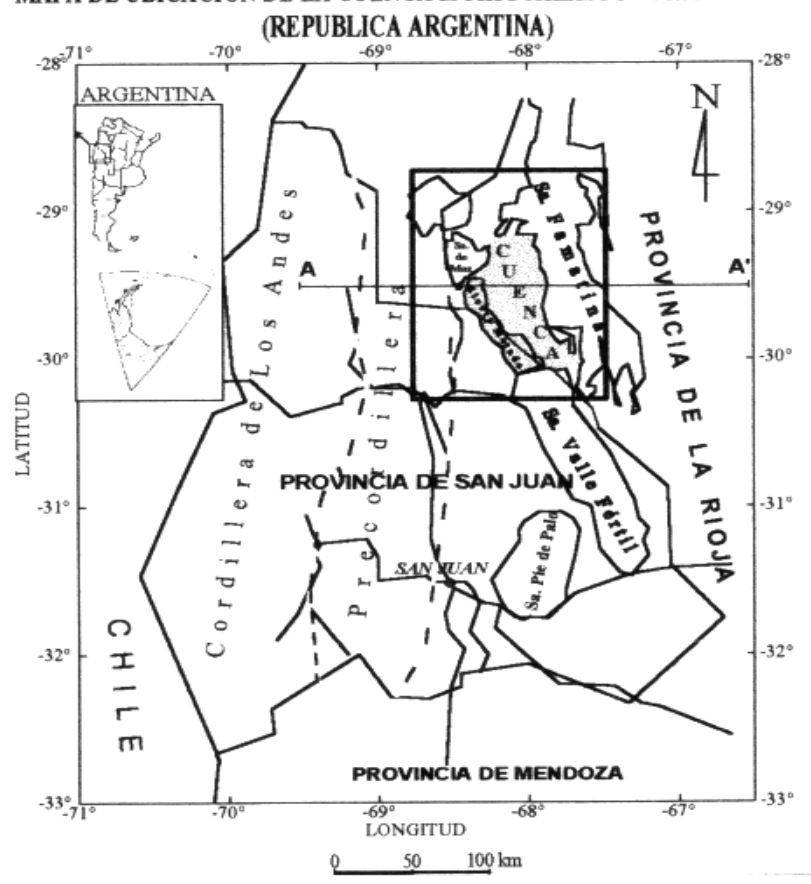

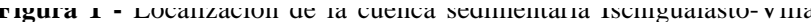
Unión, San Juan, Argentina. Área de estudio. A- -A’ Sección gravimétrica en Fig. 7.

Figure 1- Location of the Ischigualasto-Villa Unión sedimentary basin, San Juan, Argentina. Study area. A- -A' Gravity section in Fig. 7.

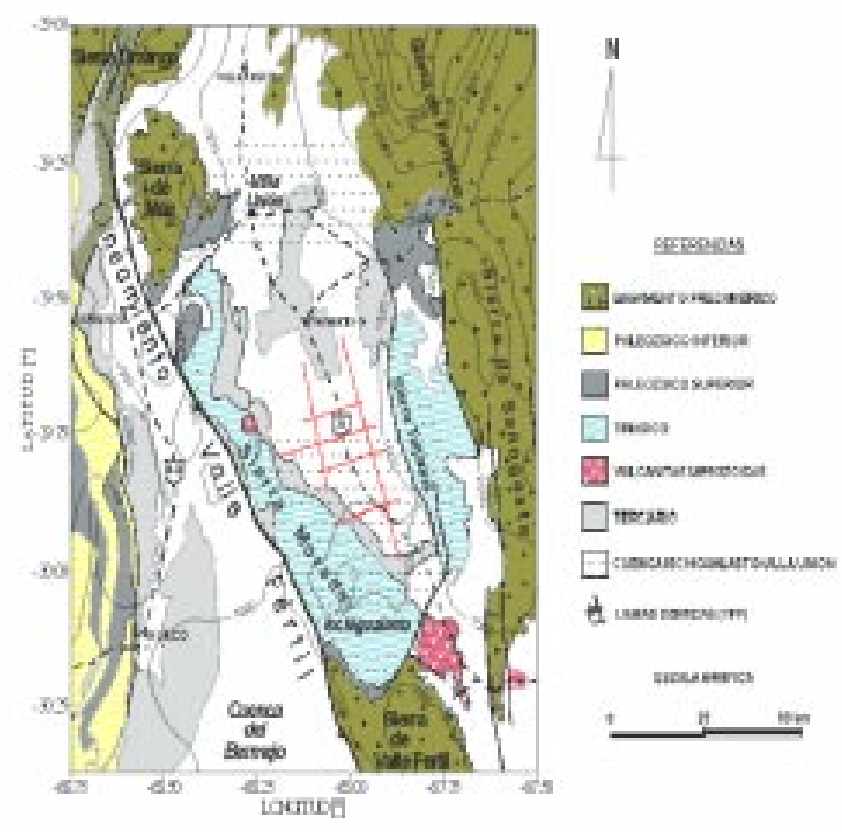

de líneas sísmicà en Fig. 11.

Figure 2- Simplified geological map of de studied region and seismic lines location in Fig. 11.

cuenca se prepararon cartas de gravedad, que adecuadamente filtradas permitieron separar la residual correspondiente al aporte gravimétrico del depósito sedimentario, a partir de ella logramos obtener la geometría de la cuenca (límites) y proponer un modelo por inversión 3D, que proporciona 9 $\mathrm{km}$ como máxima potencia sedimentaria.

\section{ADQUISICIÓN Y PROCESAMIENTO DE LOS DATOS}

Se efectuó un relevamiento gravimétrico, observándose casi 300 estaciones adecuadamente distribuidas sobre el área de la cuenca, se sumaron a ellas más de 2000 valores distribuidos en forma regional. De esta manera se conformó una grilla irregular que cubre toda la comarca (Fig. 3) permitiendo disponer, en promedio, de una estación cada $25 \mathrm{~km}^{2}$ y cuya precisión es de $1 \mathrm{mGal}$.

Los valores de gravedad se vincularon con la estación fundamental de Miguelete, Buenos Aires, en el Sistema IGSN 1971 (979690,03 mGal). El cálculo de anomalías se efectuó por las expresiones clásicas:

$\mathbf{A A L}=\mathbf{G}-(\mathrm{g}-\mathrm{CAL})$,

$A B=G^{o b}=\left(g_{0}^{0}-C A L+C B\right)$,

donde:

AAL: Anomalía de aire libre,

AB: Anomalía de Bouguer,

G : Gravedad Observada (Sistema IGSN, 1971)

g : : Gravedad Teórica Normal para el Elipsoide de 1967 o prácticamente de 1971 ,

g $[\mathrm{mGal}]=978031,85\left(1+0,0053024 \operatorname{sen}^{2} j\right.$

$$
\left.-0,0000058 \operatorname{sen}^{2} 2 j\right) \text {, }
$$

CAL: Corrección de Aire Libre, 


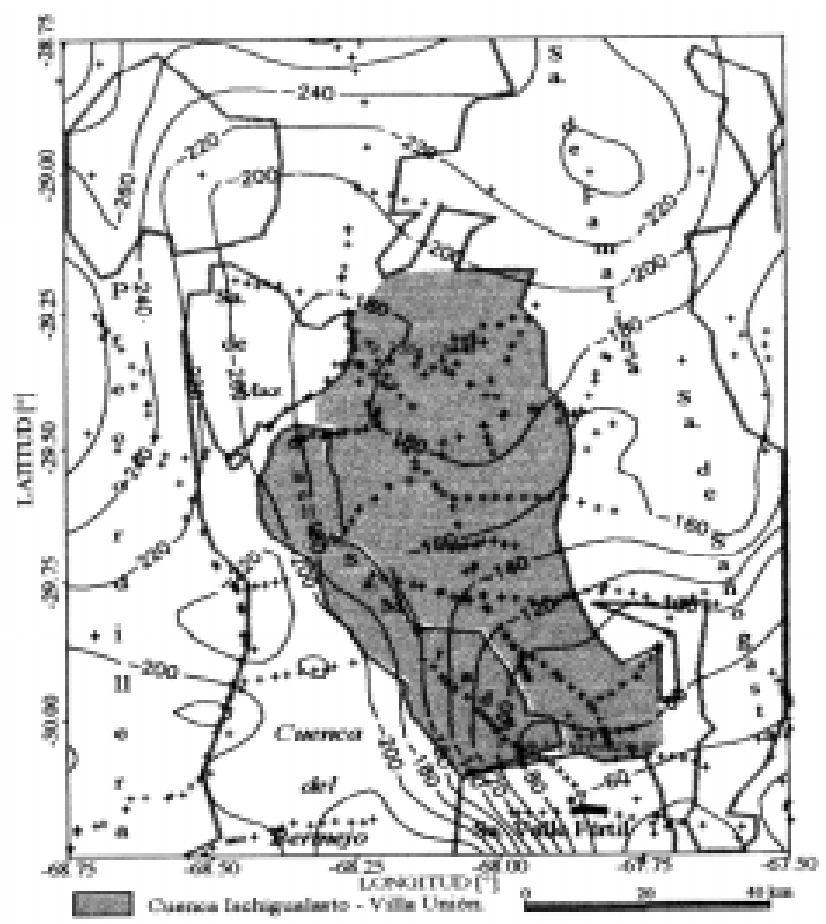

Figura 3- Carta de anomalías de Bouguer y localización de estaciones gravimétricas. Equidistancia $20 \mathrm{mGal}$

Figure 3- Bouguer anomaly map and gravity station location. Contour interval $20 \mathrm{mGal}$

CB: Corrección de Bouguer,

Para la Corrección de Aire Libre se adoptó la expresión propuesta por Swick (1942), válida para todo tipo de zonas, llanas y elevadas:

$$
\begin{aligned}
\mathrm{CAL}= & (0,308555+0,00022 \cos 2 j) \mathrm{h} \\
& -\mathbf{0 , 0 7 2}(\mathrm{h} / 1000)^{2}
\end{aligned}
$$

En tanto que para la corrección de Bouguer se asumió, como es usual, la densidad de $2,67 \mathrm{~g} / \mathrm{cm}^{3}$ :

\section{$\mathbf{C B}=\mathbf{0 , 1 1 1 8} \mathrm{h}+\mathbf{E T}$}

En (4) y (5): $\mathbf{h}$ es la altitud de la estación y en (3) y (4): $\mathrm{j}$ es su latitud.

La cuenca posee una superficie topográfica suave con $1200 \mathrm{~m}$ de altitud media respecto del nivel del mar, sin embargo, las sierras que la rodean (afloramientos de basamento cristalino) se elevan hasta $6000 \mathrm{~m}$ (Fig. 2). Por esta razón, en cada estación, se eliminó de la gravedad observada su efecto gravimétrico (ET). Este se computó por modelado directo de las sierras (Famatina, Sañogasta, Valle Fértil, Maz y Umango), utilizando el algoritmo de Talwani \& Ewing (1960), implementado por Guspí et al. (1987). Se asumió la densidad media del basamento cristalino $\left(\mathrm{s}_{\mathrm{cs}}=2,70\right.$ $\mathrm{g} / \mathrm{cm}^{3}$ ), calculada mediante relaciones velocidad-densidad, como se verá más adelante.

Los valores topográficos se obtuvieron de observaciones propias, de una red cedida por la Empresa BHP Petroleum Argentina (inédita), con precisión de $20 \mathrm{~mm} /$ $\mathrm{km}$, y complementadas (Ruiz, 1998) por cartas topográficas 1:100000 (Instituto Geográfico Militar Argentino) y geológicas 1:200000 (Servicio Geológico). La Fig. 4 muestra

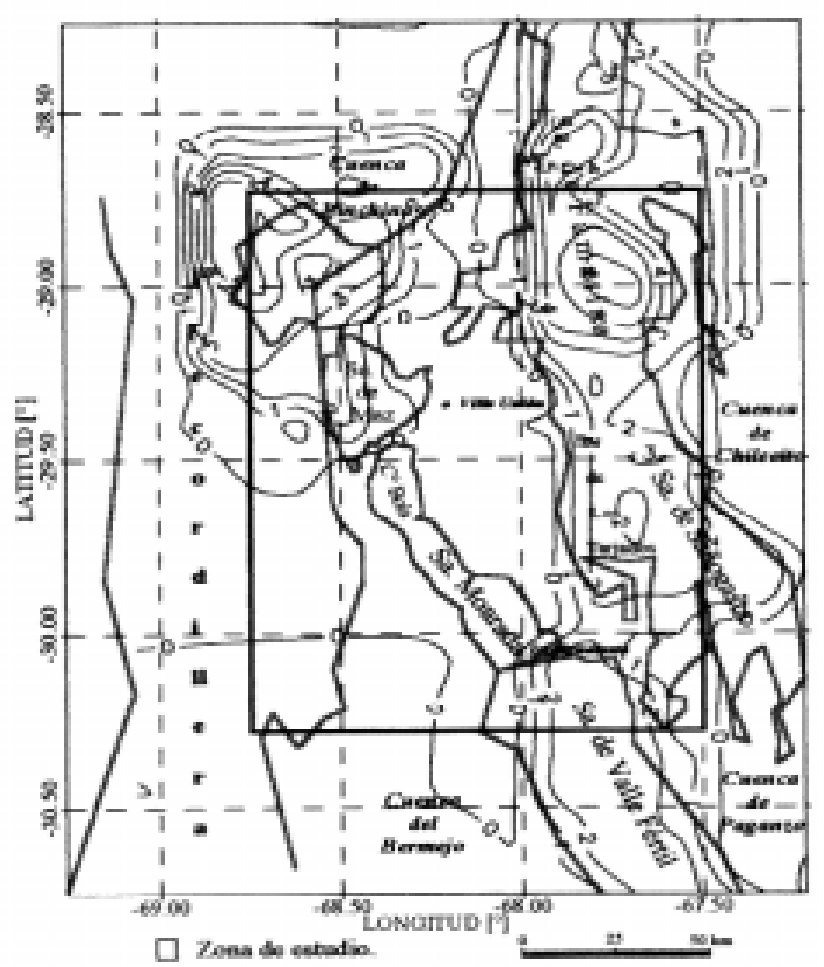

Figura 4- Carta de efectos topográficos (ET), calculados por modelado directo de las sierras que bordean a la cuenca en estudio. Equidistancia 1 mGal.

Figure 4- Topographic effects map (ET), calculated by the gravimetric inversion of the surrounding mountain range mass. Contour interval 1 mGal.

el efecto topográfico así calculado.

Las cartas de gravedad se confeccionaron regularizando la red de observaciones mediante técnicas de procesamiento digital asistidas por computadora, se utilizó el método de interpolación de mínima curvatura, apropiado para el tratamiento de campos potenciales con muestreo irregular (Briggs, 1974).

\section{Corrección geológica}

Con el propósito de normalizar la respuesta gravimétrica de la corteza superior, se efectuó una corrección geológica tentativa (Hammer, 1963; Introcaso, 1989). Esta consistió en descontar de la Carta de Anomalías de Bouguer (Fig. 3), el efecto gravimétrico calculado para las cuencas emplazadas en las vecindades de la fosa en estudio, sin incluirla. La corrección elimina la influencia de las cuencas del Bermejo, Paganzo, Chilecito y Vinchina (Fig. 5).

En primer término se calculó el efecto gravimétrico que origina el relleno sedimentario (CG) de las mismas, empleando el método de Talwani \& Ewing (1960) implementado por Guspí et al. (1984). Este se basa en integraciones numéricas establecidas en valores provenientes de un número impar de capas poligonales horizontales que conforman las isóbatas de basamento. La información se obtuvo de una carta de isóbatas de basamento sísmico en 


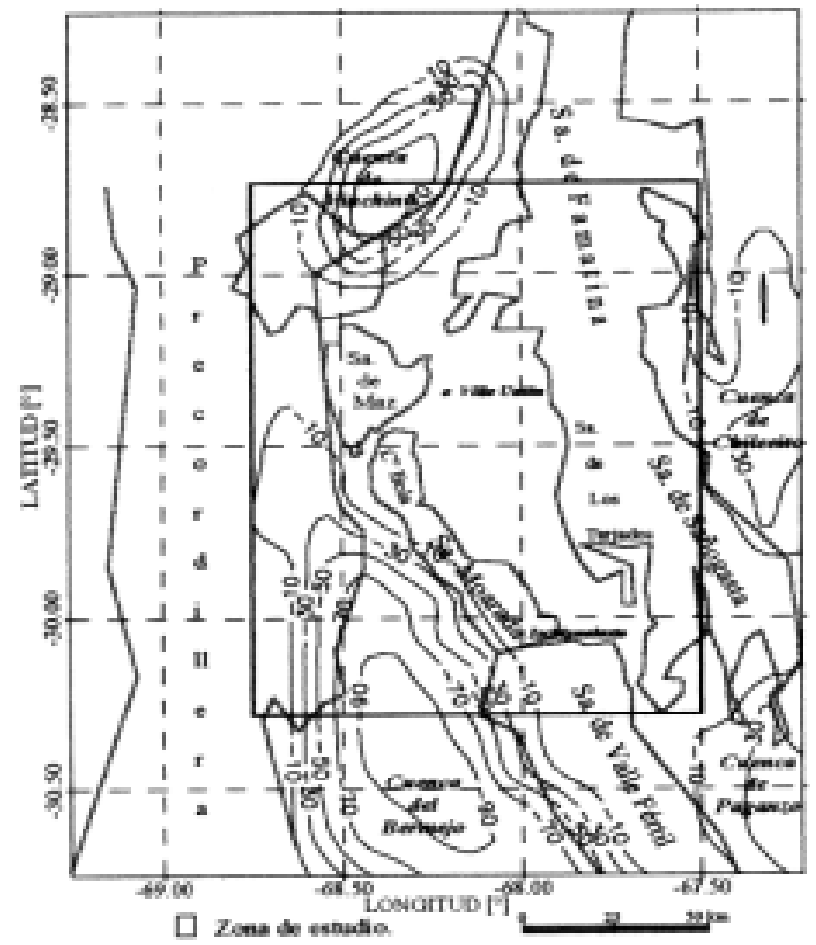

Figura 5- Carta CG, efectos gravimétricos del relleno sedimentario de las cuencas del Bermejo, Vinchina, Chilecito y Paganzo. Equidistancia $20 \mathrm{mGal}$.

Figure 5- CG map, gravity sedimentary filling effects of the Bermejo, Vinchina, Chilecito and Paganzo basins. Contour interval $20 \mathrm{mGal}$.

escala 1:2500000 (inédita) perteneciente a la Empresa Pluspetrol Argentina, ésta fue adaptada y complementada por nosotros en base a datos aislados (perfiles de refracción efectuados por Y.P.F. Sociedad del Estado y geología de superficie).

Se calculó la densidad promedio $\mathbf{s}$ de los sedimentos a partir de las velocidades de ondas compresionales extraídas de leyes de velocidad (Y.P.F., inéditas), adoptándose la relación Vp-s de Nafe \& Drake (1958); para ello, por el método de promedios pesados se obtuvieron las velocidades medias correspondientes al paquete sedimentario, $\mathrm{Vpp}=$ $4200 \mathrm{~m} / \mathrm{seg}$, y al basamento técnico, $\mathrm{Vpp}_{\mathrm{cs}}=6100 \mathrm{~m} / \mathrm{s}$, resultando las densidades $\mathbf{s}=2,44 \mathrm{~g} / \mathrm{cm}^{3} \mathrm{y} \mathrm{s}_{\mathrm{cs}}=2,70 \mathrm{~g} / \mathrm{cm}^{3}$, respectivamente.

Por último, se corrigió la carta de anomalías de Bouguer por la reducción $\mathbf{C G}$, procedimiento que puede ser sintetizado por la expresión ABcg=AB-CG. Como resultando se obtuvo una carta de gravedad, ver Fig. 6, cuya única contribución sedimentaria es la correspondiente a la cuenca en estudio, efecto que se pretende aislar a través de filtrados.

\section{SEPARACIÓN DE ANOMALÍAS}

Las anomalías observadas contienen el efecto de los cuerpos someros, intermedios y profundos, que se manifiestan en la respuesta gravimétrica como suma de cortas, medianas y largas longitudes de onda. En este caso se trata de filtrar el efecto sedimentario de una cuenca intermontana ubicada en la región límite de los terranes Precordillera y Sierras Pampeanas, zona afectada por intensa actividad tectónica, hecho que complica las tareas de separación de efectos gravimétricos. Para dar confiabilidad a los resultados se procedió de la siguiente forma:

\section{I- Filtrado por Suavización Gráfica}

El conjunto cuenca-sierras vecinas representa un máximo gravimétrico (Sierras Pampeanas Occidentales ?), ver el perfil mostrado en Fig. 7, dentro de este máximo de larga longitud de onda se encuentran las respuestas de longitud de onda intermedia de: a) Lineamiento tectónico Valle Fértil-La Huerta con el afloramiento de las sierras asociadas (Maz, Morada y Valle Fértil), que a su vez constituye el límite este de la cuenca del Bermejo; b) Cuenca Ischigualasto-Villa Unión; c) Sistema del Famatina (Sierras de Famatina, de Sañogasta y de Vilgo) y d) Sierra de Velasco, por otro lado, entre c y d se encuentra la cuenca de Chilecito.

Se empleó el método gráfico de suavización por perfiles, éste permitió discriminar los efectos asociados con las distintas fuentes (Cordillera, Precordillera, Cuencas del Bermejo e Ischigualasto-Villa Unión, Sistema de Famatina, etc.). La separación se efectuó en 12 secciones este-oeste, a intervalos de $7,5^{\prime}$ de latitud desde $29^{\circ} \mathrm{S}$ a $30^{\circ} 30^{\prime} \mathrm{S}$. Cada regional se trazó sobre las anomalías de Bouguer, aislando el efecto de la cubeta sedimentaria de cuerpos de longitud

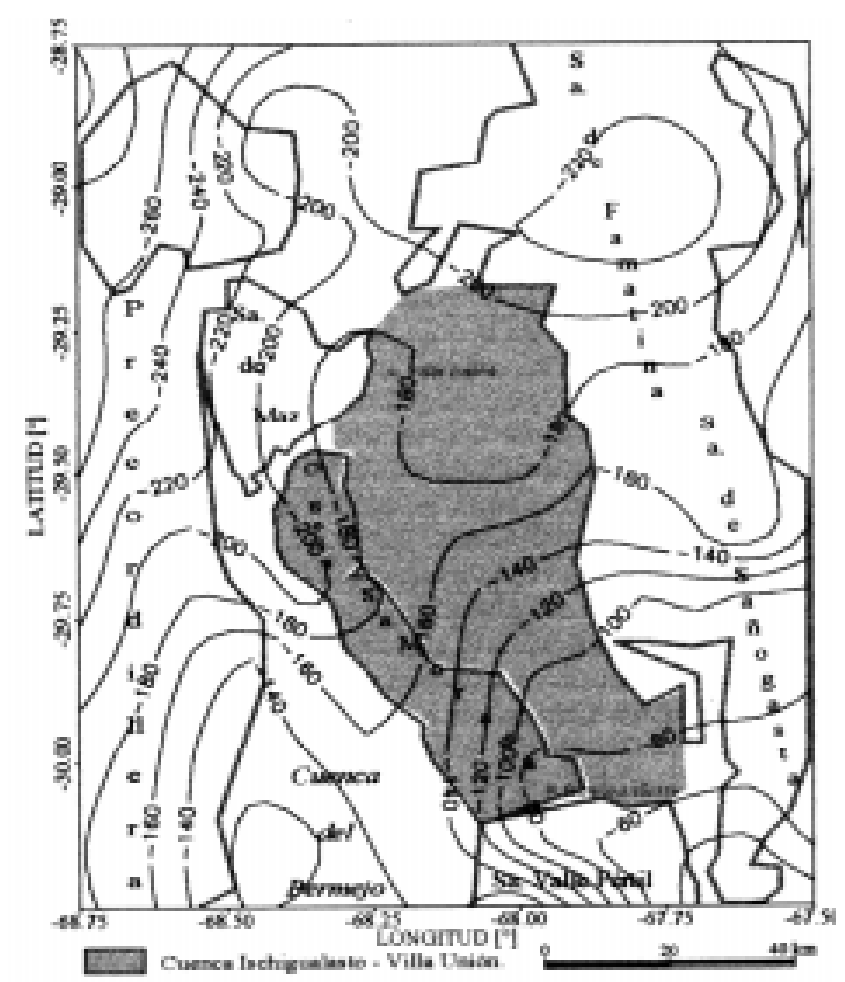

Figura 6- Carta ABcg, anomalías de Bouguer corregidas por los efectos sedimentarios CG. Equidistancia $20 \mathrm{mGal}$.

Figure 6- ABcg map, Bouguer anomaly corrected by sedimentary filling effects CG. Contour interval $20 \mathrm{mGal}$. 
de onda mayor apoyándonos en datos sísmicos esporádicos (leyes de velocidad-profundidad inéditas proporcionadas por la Empresa Y.P.F.) que fueron convertidos en anomalías de gravedad mediante el procedimiento descripto en el apartado anterior. La carta de anomalías residuales (efecto del relleno sedimentario) de la cuenca ARC (Fig. 8), se construyó por regularización de los perfiles aplicando el método de Mínima Curvatura.

\section{II- Filtrado sobre la Carta de Anomalías con Corrección Geológica}

Con el propósito de corroborar los resultados obtenidos en (I), se efectuaron filtrados numéricos sobre la carta ABcg, es decir, anomalías de Bouguer corregidas por las señales de las fosas sedimentarias vecinas a la cuenca en estudio.

A fin de obtener el efecto gravimétrico provocado por la cubeta sedimentaria, es menester encontrar la regional (AR) que elimine las influencias corticales que involucran, además, a la raíz Andina. La diferencia entre la anomalía observada y AR se interpreta como la respuesta de la corteza superior, que en ausencia de otras masas anómalas debe atribuirse al efecto de los sedimentos alojados en la cuenca.

Para el cálculo de AR se emplearon los métodos: iProlongación Ascendente de Campos Potenciales, según Pacino \& Introcaso (1987), y ii- Superficies de Tendencia (filtrado polinómico en 3-D por mínimos cuadrados, ver por ejemplo: Pacino, 1983). El software empleado fue producido por el Instituto de Física de Rosario (IFIR, U.N.R.).

La anomalía residual debida a la corteza superior (ER) se obtuvo a partir de la diferencia entre las anomalías de Bouguer y la regional: $\mathbf{E R = A B - A R}$. Los efectos residuales, resultantes de aplicar este procedimiento en (i) y (ii), se muestran en Figs. 9 y 10, respectivamente.

Recordemos que el método (i) consiste en: a- Efectuar prolongaciones ascendentes de la señal observada hasta la altura $\mathbf{h}$ en que se filtran los efectos gravimétricos superficiales; b- desde h se invierte el campo prolongado, calculando un modelo de corteza de una capa; c- la anomalía regional se obtiene computando el efecto directo de (b-) desde el nivel topográfico.

La carta ER (Fig. 9) formulada a partir de (i) es consistente, en buena medida, con la ARC (Método de Suavización), sobre todo si tenemos en cuenta la pérdida de sensibilidad de la prolongación ascendente $(\mathrm{h}=30 \mathrm{~km})$. En este punto, es importante notar que el método de separación empleado involucra efectos gravimétricos profundos, razón por la cual, la comparación entre ARC y ER (Prolongación Ascendente) permite inferir que en el sector central y norte de la cuenca, la residual responde principalmente al efecto de los sedimentos alojados en ella. En el sur de la misma, prevalecen efectos positivos en corteza atribuibles a intrusiones de rocas máficas, de las cuales hay evidencias geológicas (Entre otros, Vujovich, 1992; Page et al., 1997).

En el efecto residual resultante del filtrado por Superficies de Tendencia (Fig. 10), esta anomalía positiva es más notable, a tal punto que enmascara totalmente la respuesta sedimentaria. Como es bien conocido, el filtrado polinómico implica calcular regionales ajustando superficies de distintos órdenes al campo observado, utilizando el método de mínimos cuadrados. Las regionales así obtenidas tienden a promediar los datos de observación dando, no obstante, buenos resultados en la definición geométrica de las anómalías.

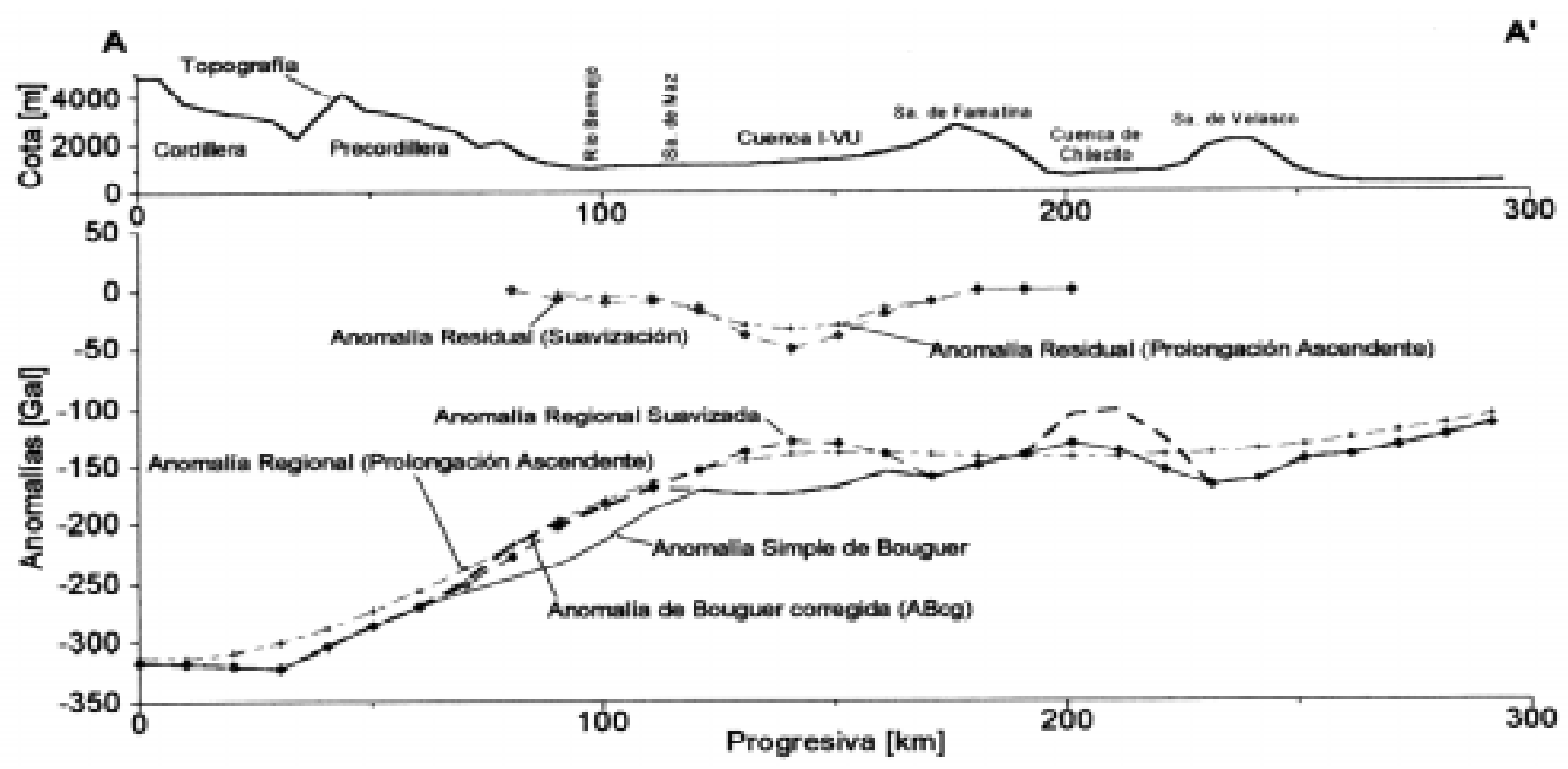

Figura 7- Perfil gravimétrico en $29^{\circ} 30^{\prime}$ de latitud sur (Fig. 1). a) Topografía; b) Anomalías de Bouguer versus anomalías regionales y residuales obtenidas por filtrados numéricos y gráficos.

Figure 7- Gravity profile 29 30' South latitude (Fig. 1). a) Topographic profile; b) Bouguer anomaly versus regional and residual anomalies derived by numerical and graphical filterings. 


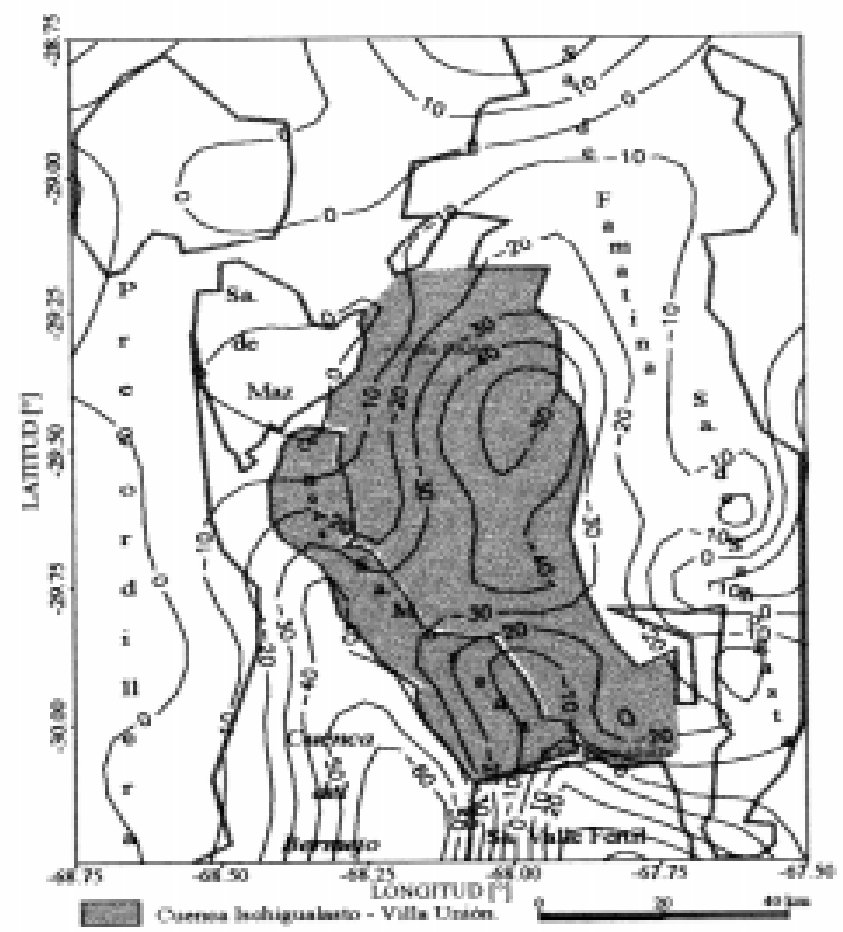

Figura 8- Carta de anomalías residuales (ARC). Efecto del relleno sedimentario separado por el método de suavización gráfica. Equidistancia $10 \mathrm{mGal}$.

Figure 8-Residual anomalies map (ARC). Sedimentary filling effect separated by graphical smoothing method. Contour interval $10 \mathrm{mGal}$.

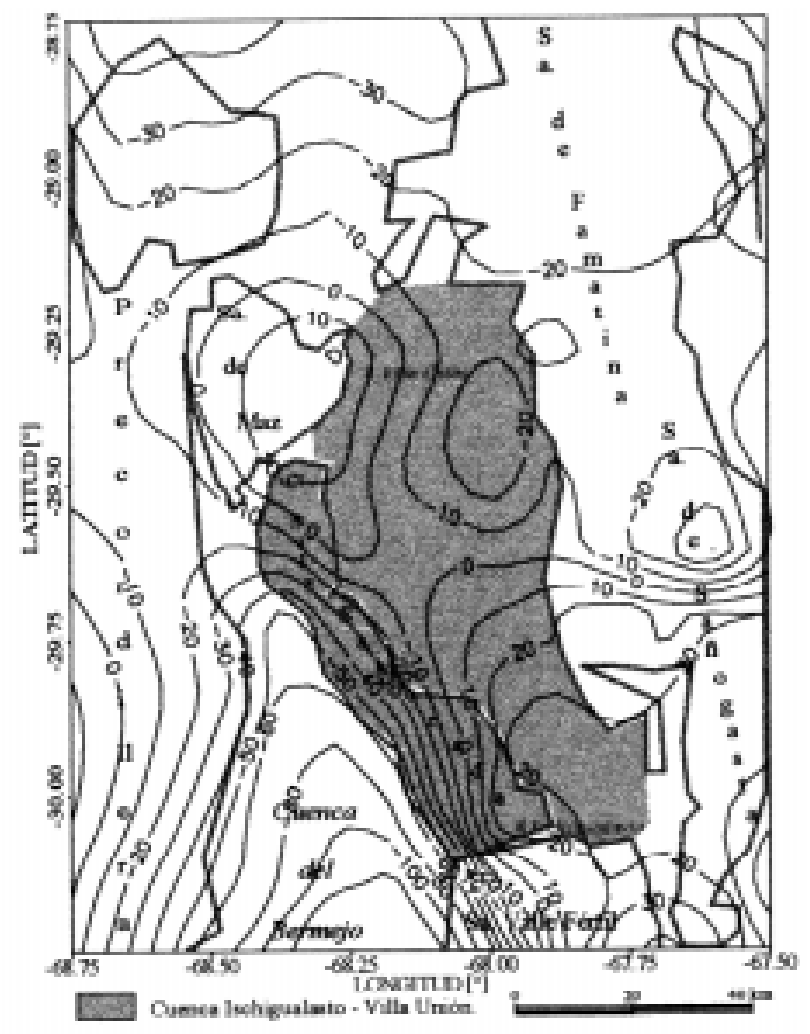

Figura 10- Efectos residuales $(\mathbf{E R}=\mathbf{A B}-\mathbf{A R})$ separados usando el método Superficies de Tendencia (polinomio de grdo 2). Equidistancia $10 \mathrm{mGal}$.

Figure 10- Residual effects $(\boldsymbol{E} \boldsymbol{R}=\boldsymbol{A B}-\boldsymbol{A} \boldsymbol{R})$ separated using least-square polynomial filtering (order 2 surface). Contour interval $10 \mathrm{mGal}$.

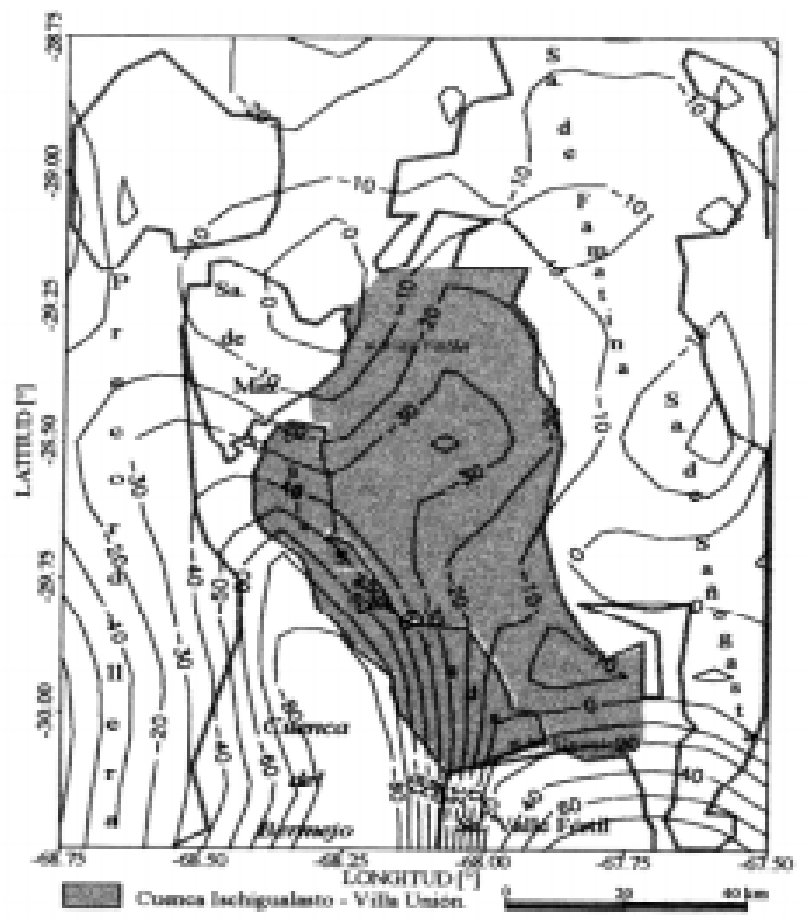

Figura 9- Efectos residuales $(\mathbf{E R}=\mathbf{A B}-\mathbf{A R})$ separados usando el método de prolongación ascendente. Equidistancia $10 \mathrm{mGal}$.

Figure 9- Residual effects ( $\boldsymbol{E} \boldsymbol{R}=\boldsymbol{A} \boldsymbol{B}-\boldsymbol{A} \boldsymbol{R})$ separated using upwards continuation method. Contour interval $10 \mathrm{mGal}$.

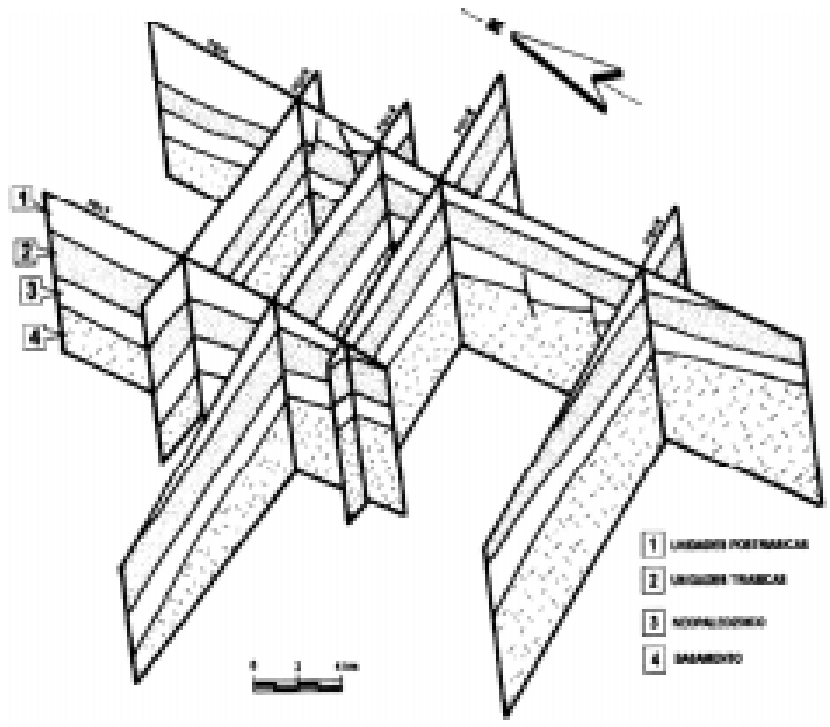

(1994), ver ubicación en Fig. 2.

Figure 11- Seismic lines interpretation by Georgieff \& Ibañez (1994), see location in Fig. 2.

\section{DISCUSIÓN}

Como veremos luego, tomando la ARC (Fig. 8) hemos definido la geometría de la cuenca por inversión gravimétrica. En esta carta puede apreciarse el gradiente negativo $(-1,5 \mathrm{mGal} / \mathrm{km})$ sobre el borde oeste de la Sierra Morada, atribuible a la falla regional Valle Fértil que pone en contacto las cuencas Ischigualasto-Villa Unión con la del Bermejo, estructura que debe ser considerada como límite 
(A)

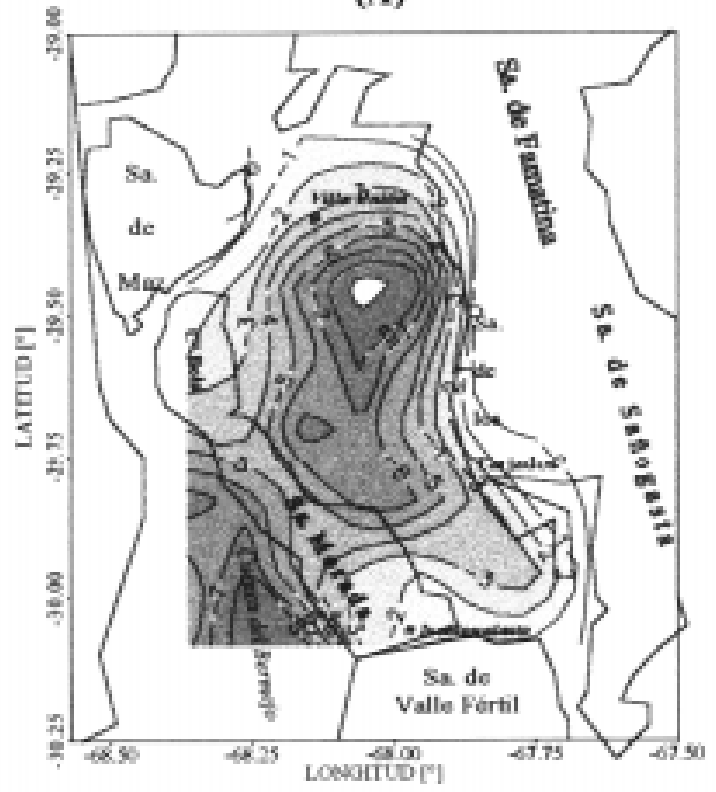

(B)

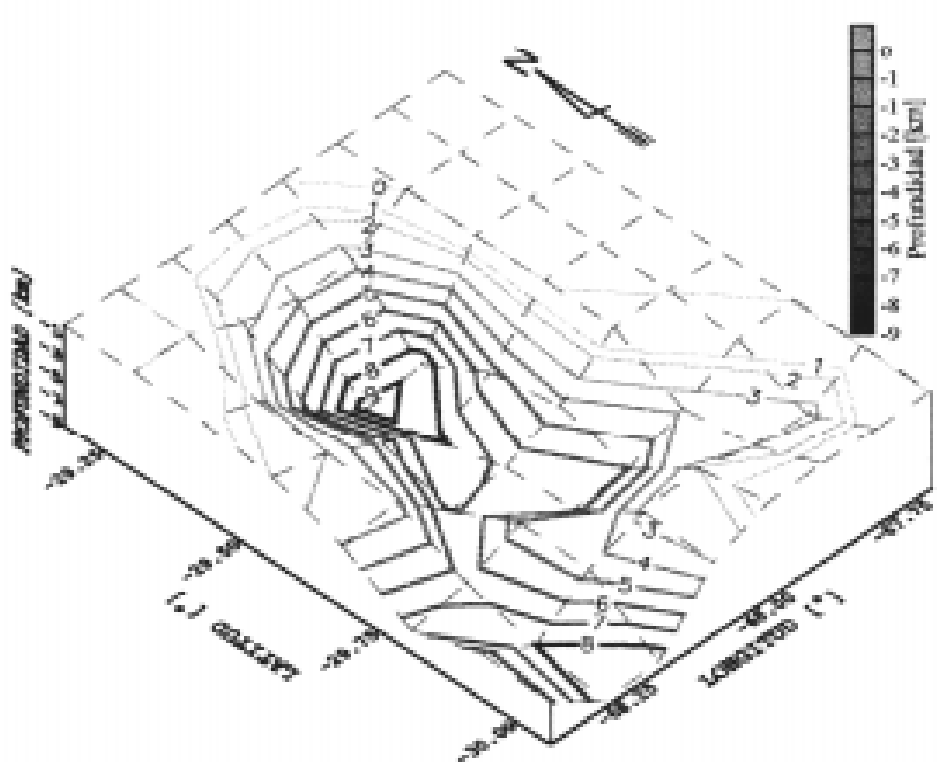

Figura 12- Modelo sedimentario 3D de una capa con $\mathrm{Ds}=-0,26 \mathrm{~g} / \mathrm{m}^{5}$. A) Isobatas de basamento, B) Vista de la superficie del basamento.

Figure 12- $3 D$ one layer sedimentary model with $D s=-0.26 \mathrm{~g} / \mathrm{m}^{3}$. A) Depth contour lines of basement, B) basement surface view.

oeste de la cubeta en estudio, por otro lado, esta falla eleva la Sierra Morada constituida por sedimentos triásicos y terciarios. Nótese que las isoanómalas negativas penetran bajo las sierras vecinas, en correspondencia con los afloramientos sedimentarios de las mismas. Los mayores espesores sedimentarios deberían desarrollarse en correlación con los mínimos gravimétricos ubicados en forma meridional.

La disminución de los valores anómalos al sudeste de la cuenca I.-V.U. es consistente con la ausencia de estratos terciarios, de mayor contraste de densidad, según secciones sísmicas (Fig. 11) publicadas por Georgieff \& Ibañez (1994) y Malizia et al. (1995). Por otro lado, puede interpretarse un aumento de la densidad del basamento cristalino por la presencia de densas rocas emplazadas en corteza media y superior (Ruiz \& Introcaso, 1997; Ruiz, 1998).

Se preparó un modelo gravimétrico a partir de la ARC, la inversión 3D se llevó a cabo empleando el método de Talwani \& Ewing (1960) según Guspí et al. (1987), es decir, la anomalía en un punto exteior a un cuerpo homogéneo, definido por secciones verticales paralelas de contorno poligonal, vinculadas lateralmente por caras triangulares, es calculada por integración analítica.

La superficie superior del cuerpo se consideró fija en $\mathrm{z}=0$, la superficie inferior (variable) fué definida por la coordenada $\mathrm{z}$ de una grilla de $8 x 8$ vértices con $D x=9,6 \mathrm{~km}$ y Dy=14 km. El modelo se computó por ensayo y error, su convergencia es del $5 \%$.

Las densidades adoptadas se obtuvieron a partir de perfiles sísmicos siguiendo la metodología descrita anteriormente, se asumió una densidad diferencial de Ds = $0,26 \mathrm{~g} / \mathrm{cm}^{3}\left(\mathrm{Ds}=2,44 \mathrm{~g} / \mathrm{cm}^{3}-2,70 \mathrm{~g} / \mathrm{cm}^{3}\right)$. Las profundidades se ajustaron con datos aislados de sísmica de reflexión y refracción (Y.P.F, inéditos), y con profundidades obtenidas por análisis espectral de la señal gravimétrica (Ruiz, 1998). Los resultados se muestran en Figs. 12a y 12b.

En el oeste, puede apreciarse el notable efecto gravimétrico de la profunda cuenca del Bermejo, expresada por anomalías de $-70 \mathrm{mGal}$, que se suma al aporte de la cuenca I.-V.U.. En la inversión debió tenerse en cuenta tal influencia, ambas cuencas se consideraron separadas por la falla regional Valle Fértil. El modelo brinda la geometría areal de la fosa y su relación con el sector norte de la cuenca del Bermejo. En el sudeste de Villa Unión, se interpretan los espesores máximos hasta el basamento técnico que son del orden de $9 \mathrm{~km}$. El volumen sedimentario calculado para la cuenca es de $1,3 \times 10^{4} \mathrm{~km}^{3}$.

El modelo propuesto en este trabajo, si bien es consistente desde el punto de vista geométrico con la interpretación de lineas sísmicas publicada por Georgieff \& Ibañez (1994) para el sector central de la cuenca, aporta espesores sedimentarios menores (en el orden del $80 \%$ ). Esta discrepancia se debe, en nuestra opinión, a que el procesamiento de las líneas sísmicas fue efectuado usando datos geológicos de afloramientos, dado que las empresas petroleras han mantenido inédita la información sísmica.

\section{CONCLUSIONES}

La metodología empleada en la separación de anomalías, permitió aislar la respuesta gravimétrica provocada por el paquete sedimentario de la cuenca intermontana Ischigualasto-Villa Unión, y por otro lado, identificar efectos anómalos positivos en el sur de la comarca, 
probablemente, provocados por cuerpos intruidos.

La consistencia de la carta de anomalías residuales ARC, obtenida por suavización gráfica, respecto del residuo ER, calculado regionalizando las anomalías corregidas por efectos sedimentarios (ABcg) y descontando tal regional a la carta de anomalías de Bouguer (AB), permite interpretar que la residual ARC, corresponde principalmente al efecto del relleno sedimentario. A partir de ésta se pudieron establecer: i- los límites de la cubeta en toda su extensión areal; ii- la distribución de los posibles espesores máximos y mínimos; iii su relación con las estructuras presentes, especialmente el importante gradiente $(-1,5 \mathrm{mGal} / \mathrm{km})$ provocado por la falla regional Valle Fértil.

Por inversión gravimétrica apoyada en datos sísmicos se preparó un modelo tridimensional, que se cree es representativo de la distribución sedimentaria de la cuenca. Empleando el contraste de densidad promedio del relleno $\left(\mathrm{Ds}=-0,26 \mathrm{~g} / \mathrm{cm}^{3}\right)$, se interpretan espesores máximos hasta el basamento técnico de $9 \mathrm{~km}$. En el modelado debió tenerse en cuenta el efecto gravimétrico causado por la cuenca vecina del Bermejo vinculada con la cubeta en estudio a través del lineamiento Bermejo-Desaguadero (falla de Valle Fértil).

\section{REFERENCIAS}

BRIGGS, I. C. - 1974 - Machine contouring using minimum curvature. Geophysics, 39: 39-48.

CAHILL, T. \& ISACKS, B. - 1992 - Seismicity and Shape of the subducted Nazca plate. J. Geophys. Res., 97:17503-17529.

DE ALBA, E. - 1954 - Descripción geológica de la Hoja 16c, Villa Unión (Provincia de La Rioja), en Boletin N 82 de la Direc. Nac. de Minería, Buenos Aires, 72 pp.

GEORGIEFF, S. - 1992 - Análisis estratigráfico del subsuelo del campo Talampaya (Cuenca Ischigualasto - Ischicuca). IV Reunión Argentina de Sedimentoloía. 2:9-16. La Rioja, Argentina.

GEORGIEFF, S. \& IBAÑNE, L. - 1994 - Principales depocentros de las supersecuencias depositacionales en el Campo de Talampaya (Neopaleozoico-Terciario). La Rioja, Argentina. V Reunión Argentina de Sedimentología. 15-19.

GUSPI, F., INTROCASO, A. \& HUERTA, E. - 1984 Calculation of gravity effects of three-dimensional structures by means of numerical integration of vertical polygonal laminae and its application to the inverse problem. Revista de Geofisica, 40:159-166.

GUSPI, F., INTROCASO, A. \& HUERTA, E. - 1987 Calculation of gravity effects of three-dimensional structures by analitical integration of a polyhedric approximation and application to the inverse problem. Geofísica Internacional, 26:407-428.

HAMMER, S. - 1963 - Deep gravity interpretation by stripping. Geophysics, 28:369-378.

INTROCASO, A. - 1989 - Procedimientos de modelado geofísico de cuencas sedimentarias enfatizando el estudio de la Cuenca San Jorge. $1^{\circ}$ Congreso Nacional de Exploración de Hidrocarburos. 605-631. Mar del Plata, Argentina.

JORDAN, T. E. - 1995 - Retroarc foreland and related basins. In Tectonics of Sedimentary Basins (Busby, C.J. \& Ingersoll, R.V. De, eds.) Blackwell Science, 331-362.

MALIZIA, D. C., REYNOLDS, J. H. \& TBBUTT, K. D. - 1995 - Cronología de la sedimentación Neógena, tectonismo y edad de la estructuración en el Campo de Talampaya, Sierras Pampeanas, Provincia de La Rioja, Argentina. YPF, BIP Junio: 78-105.

NAFE \& DRAKE - 1958 - In GRANT \& WEST - 1965 Interpretation Theory of Applied Geophysics. Mc Graw Hill Book Company. 200 pp.

PACINO, M. C. - 1983 - Determinación de anomalías regionales y residuales por medio de Superficies de Tendencia, II Simposio Sudamericano de Cogeodata, 1:151-171.

PACINO, M. C. \& INTROCASO, A. - 1987 - Regional anomaly determination using the upwards continuation method. Bollettino di Geofisica Teorica ed Applicata, XXIX(114) : 113-122.

PAge, S., LIMARINO, C. \& CASELLI, A. - 1997 Basaltos alcalinos en el Triásico de la Cuenca Ischigualasto-Villa Unión, provincias de La Rioja y San Juan. Revista de la Asociación Geológica Argentina, 52 (2):202-208.

RUIZ, F. - 1998 - Estudio de la geometría, génesis y evolución de la cuenca Ischigualasto-Villa Unión, a partir de datos de gravedad y magnetismo. Provincias de San Juan y La Rioja, Argentina. Tesis Doctoral, Universidad Nacional de Rosario.147 pp.

RUIZ, F. \& INTROCASO, A. - 1997 - Interpretación Preliminar del Efecto Gravimétrico de la Cuenca Ischigualasto-Villa Unión (Prov. de San Juan y La Rioja-Argentina). Proc. $5^{\circ}$ International Congress of the Brazilian Geophysical Society. 743-746. São Paulo, SP, Brasil.

STIPANICIC, P. \& BONAPARTE, J. - 1979 - Cuenca triásica de Ischigualasto-Villa Unión (Provincias de La Rioja y San Juan). II Simposio de Geología Regional Argentina, I:523-575. Cordoba, Argentina.

SWICK, C. H. - 1942 - Pendulum gravity measurements and isostatics reductions. U. S. Coast and Geodetic Survey. Special Publications $N^{\circ} 232$.

TALWANI, M. \& EWING, M. - 1960 - Rapid computation of gravity attraction of three-dimentional bodies of arbitrary shape. Geophysics, 25:203-225.

VUJOVICH, G. I. - 1992 - Estudio Petrológico y Tectónico del Metamorfismo del sector occidental de la Sierra de la Huerta-Provincia de San Juan. Tésis Doctoral, Universidad Nacional de La Plata.

Manuscript submitted August 24, 1998 Revised version accepted February 15, 1999 


\section{A 3D GRAVITY MODEL OF THE SEDIMENTARY DEEP BASIN OF ISCHIGUALASTO- VILLA UNIÓN (SAN JUAN AND LA RIOJA) - ARGENTINA.}

The geometry (form, extension and depth) of the sedimentary basin of Ischigualasto-Villa Unión was obtained from areal gravimetric results.

The basin is an intermontane deep graben located in a complex tectonic setting (Malizia et al., 1995; Jordan, 1995) that strongly contaminates the gravity field. For this reason, the separation of the gravimetric effect of the sedimentary filling is a complex task that it was solved through the following steps:

I- Gravity maps (Bouguer Anomalies and Bouguer Anomalies "affected by geological correction") were prepared from a properly densified data base in the basin area.

II- Through the graphical smoothing method the gravimetric effect of the sedimentary landfill of the basin in study (ARC) was separated.

III- In order to corroborate the results obtained in II, numerical filterings from the Bouguer anomalies map with geological correction $(\mathrm{ABcg})$ were made, that is to say, it was corrected by the sedimentary effects of neighboring basins and by the influence of the surrounding topographic masses. In this way, the Regional Anomaly (AR) that responds to the mass distribution at the level of the crust-mantle discontinuity was separated from $\mathrm{ABcg}$. IV- The differences among the Bouguer Anomalies (AB) and the Regional Anomalies resulting from (III) provided a residue (ER) that, in the absence of an anomalous mass at basement levels, would probably reproduce the effect of the sedimentary basin. The comparison of this residue (ER) with respect to the anomaly attributed to the basin (ARC), isolated by the graphic smoothing method, permitted to deduce that in the center and north of the basin the gravity anomalies should be mainly the result of the effect of the sedimentary filling, whereas in the south of the basin positive effects in the crust prevail, due most likely to the presence of mafic rocks located in the upper crust, corroborated by geological evidence (Vujovich, 1992; Page et al., 1997).

V- A 3D gravity model supported by seismic data that indicates sedimentary thicknesses of up to $9 \mathrm{~km}$ in the central region of the basin was prepared by using the gravimetric inversion of the residual anomaly ARC.

\section{Notas Sobre los Autores}

\section{Antonio Introcaso}

Ingeniero Geodesta-Geofísico en la Universidad Nacional de Buenos Aires (Argentina) graduado en 1962. Doctor en Ingeniería en la Universidad Nacional de Rosario (Argentina) graduado en 1997. En docencia: Profesor durante más de 30 años en 6 universidades y 8 facultades argentinas. Director de 8 Tesis Doctorales. Director de más de 30 cursos para graduados. En investigación: Investigador Principal del Consejo Nacional de Investigaciones Científicas y Técnicas (CONICET-Argentina). Director del Grupo de Geofísica del Instituto de Física de Rosario (IFIR). Autor del libro "Gravimetría”, UNR Editora, Rosario, 1997. Publicaciones en revistas: Tectonophysics, American Geophysical Union (AGU), Geoexploration (Holanda), Bolletino de Geofísica (Italia), Instituto Panamericano de Geografía e Historia (I.P.G.H.-México), Revista de Geofísica (España), Geofísica Internacional (México), RAGA (Argentina), Geoacta (Argentina), etc.. Asesorías y Referatos: O.E.A.; A.G.U. ; J. Guggenheim Memorial Fundation (New York); University of Alaska, Fairbanks; Natural Sciences and Eng. Res. Council of Canada; FAPESP (Brasil); CONICYT (Chile); CONICET (Argentina); A.A.G.G. (Argentina); RAGA (Argentina); etc. Miembro de la A.G.U.; I.A.G.; A.A.A.S.; SBGf; A.A.G.G.; RAGA. Área de Interés: Geofísica de la tierra sólida.

\section{Francisco Ruiz}

Licenciado en Geofísica en la Universidad Nacional de San Juan (UNSJ, Argentina) graduado el 3/7/92. Doctor en Ingeniería en la Universidad Nacional de Rosario (Argentina) graduado el 19/3/98. Investigador (becario) del Consejo Nacional de Investigaciones Científicas y Técnicas (CONICET-Argentina) desde 1/4/93. Docente (Profesor Ordinario Jefe de Trabajos Prácticos) en el Instituto Sismológico Ing. Fernando S. Volponi (UNSJ) desde 1/10/93 y en la cátedra Gravimetría y Magnetometría del Departamento de Geofísica (UNSJ) desde 1/4/98. Miembro de la Asociación Argentina de Geofísicos y Geodestas (A.A.G.G.). Área de interés: Campos Potenciales en estudios de corteza y litosfera terrestres. 